\title{
ANALYTIC HIERARCHY PROCESS (AHP) E A DEFINIÇÃO DE ATRIBUTOS PARA APOIO A TOMADA DE DECISÃO: UMA ANÁLISE BIBLIOMÉTRICA
}

\author{
ANALYTIC HIERARCHY PROCESS (AHP) AND ATTRIBUTE DEFINITIONS TO SUPPORT \\ DECISION MAKING: A BIBLIOMETRIC ANALYSIS
}

\section{Wellington Gonçalves ${ }^{1 *}$, Alessandro Roberto Rocha ${ }^{2}$, Fernando Nascimento Zatta ${ }^{3}, \&$ Diego Vieira dos Santos ${ }^{4}$}

${ }^{1}$ Centro Universitário Norte do Espírito Santo (CEUNES) da Universidade Federal do Espírito Santo (UFES).

${ }^{2}$ Instituto de Ciências da Vida da Universidade Federal de Juiz de Fora (UFJF).

${ }^{3}$ Centro de Ciências Sociais Aplicadas da Universidade Presbiteriana Mackenzie.

${ }^{4}$ Instituto Federal do Espírito Santo (IFES) - Campus São Mateus.

${ }^{1 *}$ wellington.goncalves@ufes.br ${ }^{2}$ alessandro.rocha@ufjf.edu.br ${ }^{3}$ zatta@hmzconsulting.com.br $\underline{{ }^{4} \text { diego.santos@ifes.edu.br }}$

\section{ARTIGO INFO.}

Recebido em: 28.09.2021

Aprovado em: 11.11.2021

Disponibilizado em: 17.11.2021

Palavras-chave:

Acoplamento bibliométrico; Bibliometria; Cocitação; Coocorrência; Web of Science.

\section{KEYWORDS:}

Bibliometric coupling; Bibliometrics; Cocitation; Cooccurrence; Web of Science.

*Autor Correspondente: Gonçalves, W.

\section{RESUMO}

Devido à sua elevada flexibilidade e ampla aplicabilidade, diferentes caminhos percorridos e pontos de vista sobre o Analytic Hierarchy Process (AHP) têm sido estudados extensivamente na literatura nos últimos 50 anos. No momento em que este trabalho foi escrito, não foi identificada uma análise bibliométrica sobre o estado da arte de como critérios estão sendo definidos e mensurados no uso do AHP para gestão de atividades e operações. Assim, realizamos uma revisão na base de dados Web of Science (WoS), utilizando artigos de pesquisa aplicada, publicados entre 1970 e 2020. Aspectos como citação e estrutura de publicação foram usados para organizar a realização da estima da evolução literária por meio do software Zotero. Em seguida, empregamos o software VOSviewer para mapear cocitação e coocorrência. Os resultados mostram um aumento gradual na estrutura de citação e publicação, além de usos e aplicações híbridas, destacando o AHP como um importante elemento para a academia e, também, para o avanço do conhecimento sobre auxílio à tomada de decisão. Este trabalho contribui com a ciência ao identificar práticas de uso do AHP, auxiliando diferentes interessados no tema a compreender os diversos caminhos e enfoques dados às vertentes de uso.

\begin{abstract}
Due to its high flexibility and wide applicability, different paths taken and points of view about the Analytic Hierarchy Process (AHP) have been extensively studied in the literature over the last 50 years. At the time this work was written, a bibliometric analysis on the state of the art of how criteria are being defined and measured in the use of AHP for managing activities and operations has not been identified. Thus, we performed a review in the Web of Science (WoS) database, using applied research articles, published between 1970 and 2020. Aspects such as citation and publication structure were used to organize the realization of the estimate of literary evolution through the software Zotero. We then employ VOSviewer software to map cocitation and cooccurrence. The results show a gradual increase in citation and publication structure, in addition to hybrid uses and applications, highlighting AHP as an important element for academia and also for the advancement of knowledge about aiding decisionmaking. This work contributes to science by identifying practices in the use of AHP, helping different people interested in the subject to understand the different paths and approaches given to the aspects of use.
\end{abstract}



atributos para apoio a tomada de decisão: uma análise bibliométrica. Brazilian Journal of Production Engineering, 7(5), 103-118.

\section{INTRODUÇÃO}

As organizações na contemporaneidade estão cada vez mais submetidas a pressões competitivas, regulatórias e comunitárias, o que tem ampliado a importância de tomada de decisões assertivas para manter equilíbrio entre os desempenhos econômico e ambiental (Santos, Neves, Sant'Anna, Oliveira, \& Carvalho, 2019). Corroborando com esta opinião, Petruni et al. (2019) destacam que associada a essa necessidade há também uma contínua demanda por escolha de alternativas que auxiliem à prescrição de soluções frente à diversidade de cenários e stakeholders.

Além disto, considerando ainda que o referido contex to se mostra suscetível a instabilidades de fatores globais, é legítimo o entendimento de que a participação de stakeholders se mostra preponderante no planejamento e operacionalização de atividades, visto que estas refletem diretamente no gerenciamento dos custos de produção, além de sofrer influência de oscilações de mercado (Friendly, 2019; Martins et al., 2017).

Nesse sentido, o ambiente competitivo que impõe margens de lucro reduzidas, altas expectativas de consumidores por produtos de qualidade e prazos curtos, segundo Yadav e Sharma (2016), levam empresas a terem que aproveitar oportunidades que surgem em seu cotidiano para otimizar seus processos de negócios. De acordo com estes autores, para atingir esse objetivo e se manterem competitivas, as organizações devem, portanto, trabalhar junto de seus principais parceiros e demais stakeholders da cadeia de suprimentos.

Para Webb (2018) o universo organizacional envolve além de contextos ambientais e atores, incertezas que na maioria das vezes estão associadas ao emprego de soluções empíricas no auxílio à tomada de decisão. Isso, para o cotidiano das organizações não é o suficiente, segundo Zhou e Yang (2020), que ainda destacam a existência da multiplicidade de cenários que influenciam as decisões relacionadas às atividades e operações, possuem ainda diversos atores que necessitam ter suas expectativas, necessidades e julgamentos ponderados por métodos e técnicas de forma a convergir opiniões.

De acordo com a literatura científica (Ho \& Ma, 2018; Merigó \& Yang, 2017; Qaiser, Ahmed, Sykora, Choudhary, \& Simpson, 2017; Santos et al., 2019), para contextos organizacionais que demandem tomadas de decisão que envolvam elevado número de atributos (critérios e subcritérios), a utilização de Multi-Criteria Decision-Making (MCDM) têm sido amplamente usados. Dentre os métodos que apoiam a tomada de decisão, o Analytic Hierarchy Process (AHP) é o mais utilizado e conhecido (Saaty, 2008; Ho \& Ma, 2018), é um método MCDM que emprega em seu desenvolvimento operacional comparações paritárias de atributos, com base em uma escala numérica, a qual sistematiza e estrutura o processo de tomada de decisão (Demuner, Zatta, Freitas, \& Goncalves, 2020).

Trabalhos como de Muralidharan, Anantharaman e Deshmukh (2002) propuseram um procedimento baseado no AHP em cinco passos para auxiliar tomadores de decisão na classificação e seleção de fornecedores, o qual contou com nove atributos de avaliação. Para realizar os julgamentos, estes autores utilizaram colaboradores de diferentes funções da unidade de pesquisa, como compras, lojas e controle de qualidade, os quais compuseram o processo de seleção. Chan e Chan (2004) utilizaram o AHP para avaliar e selecionar fornecedores. No 

atributos para apoio a tomada de decisão: uma análise bibliométrica. Brazilian Journal of Production Engineering, 7(5), 103-118.

trabalho destes autores, a estrutura hierárquica do AHP foi composta por seis critérios de avaliação com 21 subfatores, dos quais as ordenações de importância foram obtidas com base em requisitos do cliente. Bruno, Esposito, Genovese e Passaro (2012) sugeriram uma abordagem com base numa estrutura, a qual continha 12 subcritérios considerados em quatro critérios, quais sejam, qualidade do processo e produto, nível de serviço, gestão e inovação e, posição financeira para escolha de fornecedores em ambiente corporativo. A análise do processo de implementação dessa abordagem proporcionou identificar pontos fortes e fracos do uso de modelos para seleção de fornecedores e, a partir disso, apresentar auxílio à tomada de decisão para o problema de avaliação destes, indicando barreiras que limitam as organizações adotar tais soluções.

Existem diversas vertentes do uso do AHP para apoio a tomada de decisão na literatura. De fato, o AHP é altamente sensível ao contexto, pois suas interpretações podem significar fatos diferentes para pessoas e cenários desiguais (Zhou \& Yang, 2020). Dada essa situação, permitir a visualização de caminhos e possiblidades no emprego do AHP é algo que amplia horizontes, bem como, contribui para que as tomadas de decisões sejam mais céleres e eficazes nos níveis estratégico, tático e operacional (Santos et al., 2019).

Este trabalho, portanto, teve por objetivo entender como critérios estão sendo definidos e mensurados no uso do Analytic Hierarchy Process (AHP) para gestão de atividades e operações. E, com isso, ser uma tentativa de realizar uma revisão sistemática da literatura sobre o AHP e seu apoio à tomada de decisão na gestão de atividades e operações, de forma que revele o estado existente da pesquisa nesta área e forneça a pesquisadores, profissionais, gestores e organizações conhecimento sobre o estado da arte do tema, como também, uma direção para desenvolver ainda mais este campo.

\section{REFERENCIAL TEÓRICO}

Devido à sua ampla flexibilidade e extensa aplicabilidade, as abordagens do método multicritério para auxílio à tomada de decisão Analytic Hierarchy Process (AHP) têm sido aplicadas e estudadas nos últimos 20 anos (Ho \& Ma, 2018; Santos et al., 2019). Este trabalho, como um estudo de acompanhamento revisa a literatura sobre as abordagens integradas do AHP e aplicações, averiguando como critérios estão sendo definidos e mensurados em seu uso para gestão de atividades e operações.

O AHP tem por fundamento empregar o método cartesiano de pensar voltado à verticalização da mente humana durante a concepção e estruturação de um problema, com isso, variadas situações são decompostas e desmembradas em critérios, subcritérios e alternativas (Zatta, Mattos, Oliveira, Freitas, \& Gonçalves, 2019). Gonçalves (2016) destaca que o método AHP pode distinguir e analisar inconsistências em julgamentos e, a partir disso, minimizar a incidência de possíveis erros na interpretação de resultados, para tanto se apoia em três etapas. Inicialmente, segundo Saaty (2008) o problema a ser estudado deve ser modelado em níveis hierárquicos, primeira etapa, sendo considerado o objetivo a ser atingido, seguido dos critérios e subcritérios definidos conforme a conveniência e necessidade da ocasião, e das alternativas de decisão (Figura 1).

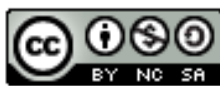



atributos para apoio a tomada de decisão: uma análise bibliométrica. Brazilian Journal of Production Engineering, 7(5), 103-118.

Figura 1. Estrutura hierárquica para operacionalização do AHP

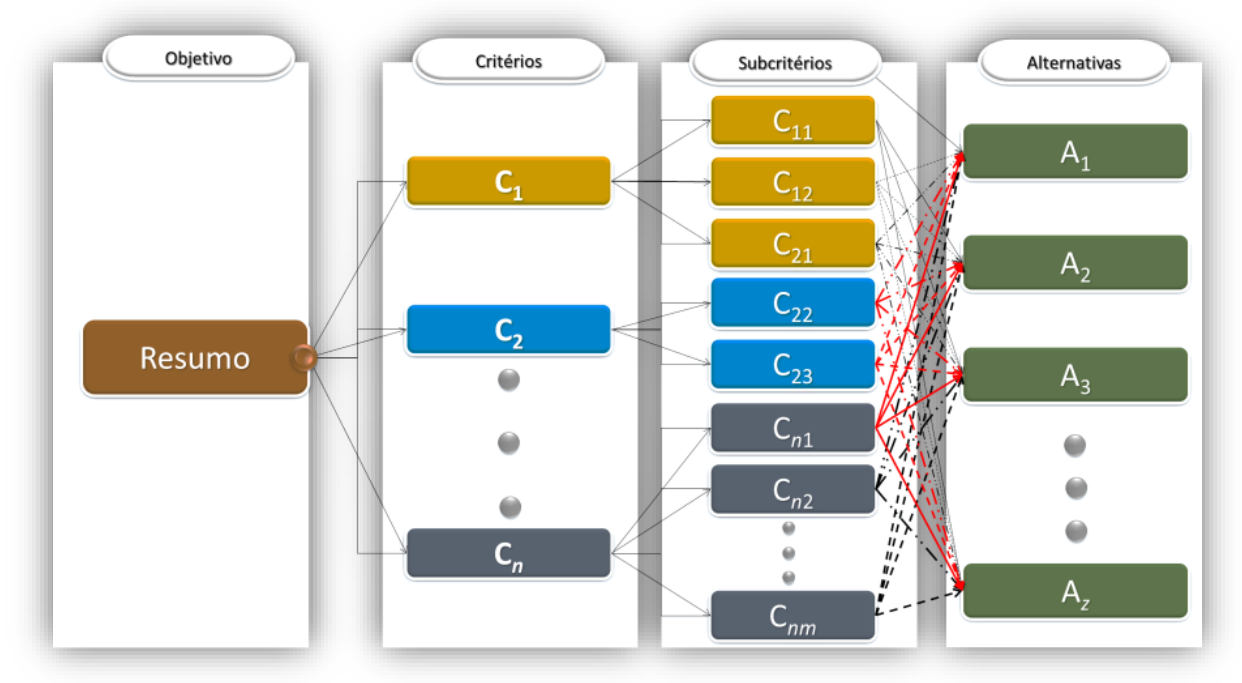

Fonte: Saaty (2008).

Posteriormente, segunda etapa, realizam-se julgamentos paritários com base na escala proposta por Saaty (2008) disposta no Quadro 1, ou seja, os critérios e subcritérios são comparados paritariamente, sendo os julgamentos efetuados com base nessa escala numérica estabelecida para ajustar consequentes resultados das percepções dos respondentes.

Quadro 1. Escala numérica para uso do método AHP

\begin{tabular}{|cl|}
\hline Intensidade da importância & \multicolumn{1}{c|}{ Definição } \\
\hline 1 & Elementos são de igual importância \\
3 & Moderada importância de um elemento \\
5 & Forte importância de um elemento \\
7 & Importância muito forte de um elemento \\
9 & Extrema importância de um elemento \\
$2,4,6$ e 8 & Valores intermediários entre as opiniões adjacentes \\
Incremento 0,1 & Valores intermediários na graduação de 0,1 \\
\hline
\end{tabular}

Fonte: Saaty (2008).

Dentro desse contexto, de acordo com Saaty (2008), uma matriz de julgamentos genérica $A$ pode ser elaborada por meio da Equação 1:

$\frac{n(n-1)}{2}$

Em que: $n$ é o número de elementos contidos na matriz $A=\left[a_{i j}\right]_{n x n}$, assim definida (Equação 2):

$A=\left[\begin{array}{cccc}1 & a_{12} & \cdots & a_{1 n} \\ 1 / a_{21} & 1 & \cdots & a_{2 n} \\ \vdots & \vdots & \cdots & \vdots \\ 1 / a_{n 1} & 1 / a_{n 2} & \cdots & 1\end{array}\right]$ 

atributos para apoio a tomada de decisão: uma análise bibliométrica. Brazilian Journal of Production Engineering, 7(5), 103-118.

Em que:

$a_{i j}>0 \rightarrow$ positiva $; a_{i j}=1 \therefore a_{j i}=1 ; a_{i j}=1 / a_{j i} \rightarrow$ recíproca $; a_{i k}=a_{i j} \cdot a_{j k} \rightarrow$ consistência

Contudo, é importante observar que a liberdade provida pelo método AHP para definição de critérios, subcritérios e alternativas pode levar a geração de inconsistências nas avaliações dos julgamentos paritários (Gonçalves, 2016). Sendo assim, Saaty (2008) propôs um valor máximo de consistência para que os julgamentos sejam considerados coerentes, terceira etapa, obtido por intermédio da Equação 3.

$$
R C=I C / I R
$$

Para determinação da Razão de Consistência $(R C)$ considera-se o Índice de Consistência Randômica $(I R)$, o qual consiste em julgamentos aleatórios para matrizes de proporções variadas (Gonçalves, 2016), e o Índice de Consistência (IC) que mede a coerência de julgamentos com base na Equação 4 (Saaty, 1977).

$$
I C=\left(\lambda_{\text {máx. }}-n\right) / n-1
$$

Saaty (1977) cita que $\lambda_{\text {máx. é o autovalor máximo resultante da multiplicação da matriz original }}$ pelo autovetor máximo obtido após o procedimento de normalização da matriz de comparação e, $n$ é considerado como o número de critérios ou subcritérios calculados. Complementarmente, o autor aponta que os julgamentos somente serão considerados confiáveis caso apresentem $R C$ menor ou igual a 0,10 (Saaty, 2008). Na opinião de Gonçalves (2016), em não atendimento a esta indicação deve ser feita uma revisão e, se for necessário, reformulação dos julgamentos, contudo, na ocorrência de nova inconsistência a estrutura hierárquica deve ser revista ou refeitos os julgamentos.

\section{AbORDAGEM Metodológica}

Para operacionalização da abordagem metodológica, dados de artigos que utilizam critérios no uso do AHP para gestão de atividades e operações foram recuperados da Coleção Principal da base de dados Web of Science (WoS) - Clarivate Analytics com uso da plataforma Periódicos Capes. Desta forma, a pesquisa foi realizada considerando parâmetros de consulta a partir dos indicativos da própria literatura. Os dados foram coletados em junho de 2021, considerando um período temporal a partir de 1970 até o ano de 2020, a partir de relevantes expressões selecionadas da literatura, as quais balizarão tal pesquisa.

A WoS foi selecionada como base de dados para auxílio à pesquisa nesse trabalho por ter uma plataforma baseada na tecnologia da Web criada em 1960, contendo um arcabouço que engloba literaturas em qualquer disciplina do conhecimento; científico, tecnológico, humanístico e sociológico desde 1945, com mais de 12.000 periódicos, e cerca de 760 milhões de fontes de referências citadas (Sánchez, Del Río, \& García, 2017). Desta forma, a abordagem metodológica foi realizada considerando 6 etapas (Figura 2). 

atributos para apoio a tomada de decisão: uma análise bibliométrica. Brazilian Journal of Production Engineering, 7(5), 103-118.

Figura 2. Síntese operacional da abordagem metodológica

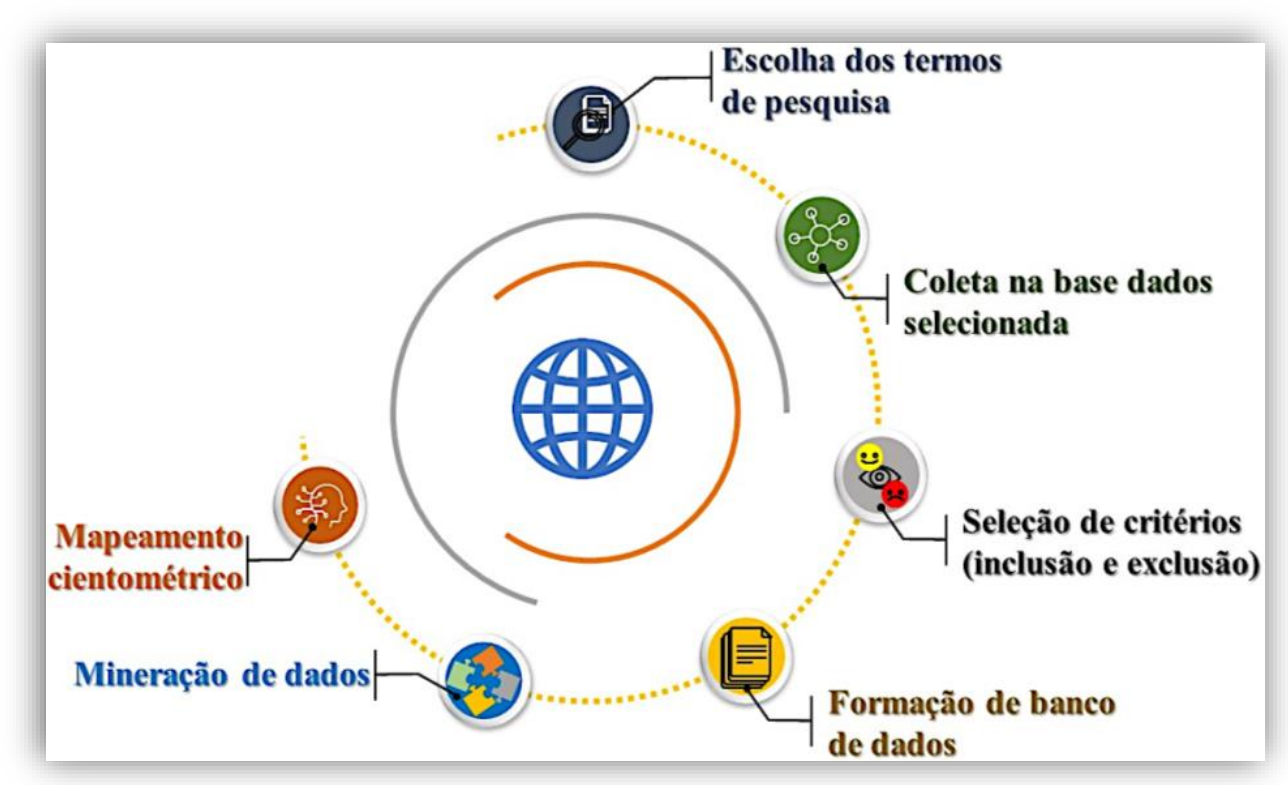

Fonte: Autores, 2021.

Desta forma, considerando o trabalho de Gora (2019) foram levantados na literatura termos considerando o uso do AHP com critérios relacionados a gestão de atividades e operações, para serem utilizados como palavras-chave. Para tanto, esses termos foram levantados por meio de consulta a WoS, ao ser utilizado o software VOSviewer versão 1.6.17, lançado em 22 de julho de 2021, para auxílio à seleção destes termos por meio de análise de redes de relacionamentos e, o software Wordaizer versão 3.6.0.116 (licença freeware), lançado em 23 de junho de 2016,que auxiliou na elabora de uma Word Cloud (primeira etapa).

O software VOSviewer foi selecionado para ser utilizado neste trabalho devido seu foco na visualização de relações de redes bibliométricas e, também possuir uma interface de usabilidade autoexplicativa em termos de recursos. Dentro desse contexto, o software Wordaizer foi utilizado em razão da sua elevada disponibilidade de escolha de efeitos, sobreposições, adesivos e bordas, os quais, inclusive podem agregar modos de mesclagem, além de permitir uma análise quantitativa de fenômenos e documentos.

Na sequência, após a identificação dos termos de pesquisa, ocorreu a segunda etapa com a realização da coleta de artigos na base de dados WoS. Uma triagem de dados foi feita a partir de critérios de inclusão e exclusão definidos, terceira etapa, a fim de retirar elementos que não estejam alinhados com os parâmetros do trabalho (Qaiser et al., 2017).

Assim, foi parametrizado e formado um banco de dados que auxiliou as atividades operacionais de análise bibliométrica (Santos et al., 2019), sendo o mesmo em seguida, adequado para colaborar com o prosseguimento da abordagem metodológica (quarta etapa). Para apoiar essa adequação foi utilizado o software Zotero. A partir deste banco, uma mineração de dados foi feita para examinar o conteúdo obtido e, a partir disso, verificar a existência de padrões, sendo empregado o software VOSviewer para auxiliar na operacionalização dessa mineração (quinta etapa). A partir dos resultados obtidos foi realizada uma análise de dados e, concomitantemente a isso, a elaboração de um mapeamento cientométrico (Gora, 2019) - sexta etapa. 

atributos para apoio a tomada de decisão: uma análise bibliométrica. Brazilian Journal of Production Engineering, 7(5), 103-118.

\section{ReSUltados}

Para realizar a análise bibliométrica, foi feita uma busca preliminar na base de dados WoS empregando palavras de contexto por meio de operadores booleanos: "TI=(Analytic Hierarchy Process OR AHP)", objetivando identificar termos para serem utilizados como palavras-chave, considerando artigos em periódicos publicados na língua inglesa no período entre 1970 e 2020. Assim, as buscas retornaram um conjunto de 4337 artigos, os quais por meio de análise junto ao software VOSviewer e, do Wordaizer foi possível construir uma Word Cloud (Cebrino \& Portero De La Cruz, 2020) a partir de 20 termos com maior incidência nesse conjunto (Figura $3)$.

Figura 3. Word cloud de termos de pesquisa sobre o uso do AHP

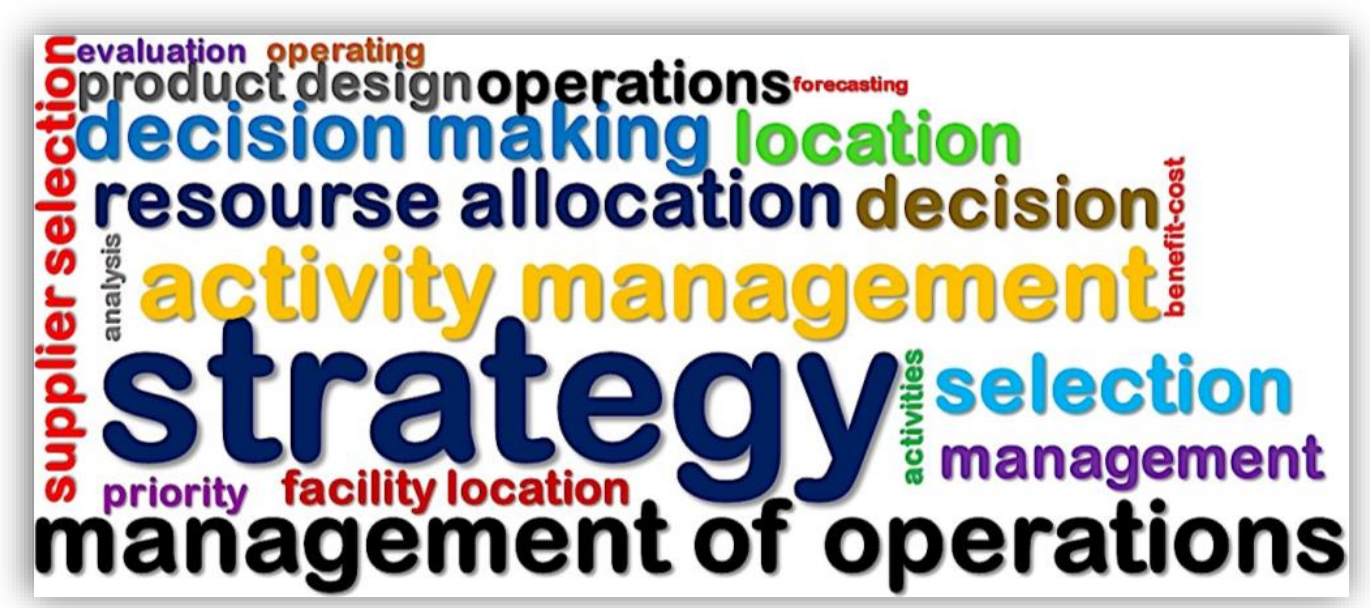

Fonte: Wordaizer.

A partir da definição dos termos (Figura 3) uma nova busca foi realizada na WoS considerando as mesmas parametrizações iniciais, porém, com a adição dos termos selecionados por meio de operadores booleanos com os rótulos de campo título, resumo e todas as palavras-chave (segunda etapa). O resultado desta busca possibilitou localizar 1076 artigos.

Prosseguindo, uma triagem de dados foi realizada na WoS utilizando operadores booleanos. Essa triagem ocorreu a partir da inclusão de artigos que continham em seu título as palavras "Analytic Hierarchy Process" ou "AHP" e, no resumo: "management"; "activities'; "operations"; "operating"; "activity management"; "management of operations"; "analysis"; "decision"; "decision making"; "selection"; "supplier selection"; "evaluation"; "priority"; "resourse allocation"; "benefit-cost"; "forecasting"; "location"; "facility location"; "product design"; "strategy", serem de acesso aberto e estarem inclusos nas categorias management; operations research management science e engineering industrial. Com isso, foram excluídos àqueles artigos que não atenderam a essa definição (terceira etapa), permanecendo ao final um total de 109 artigos (Figura 4). 
Citação (APA): Gonçalves, W., Rocha, Ar. R., Zatta, F. N., \& Santos, D. V. (2021). Analytic Hierarchy Process (AHP) e a definição de atributos para apoio a tomada de decisão: uma análise bibliométrica. Brazilian Journal of Production Engineering, 7(5), 103-118.

Figura 4. Consolidado de registros por categorias da WoS

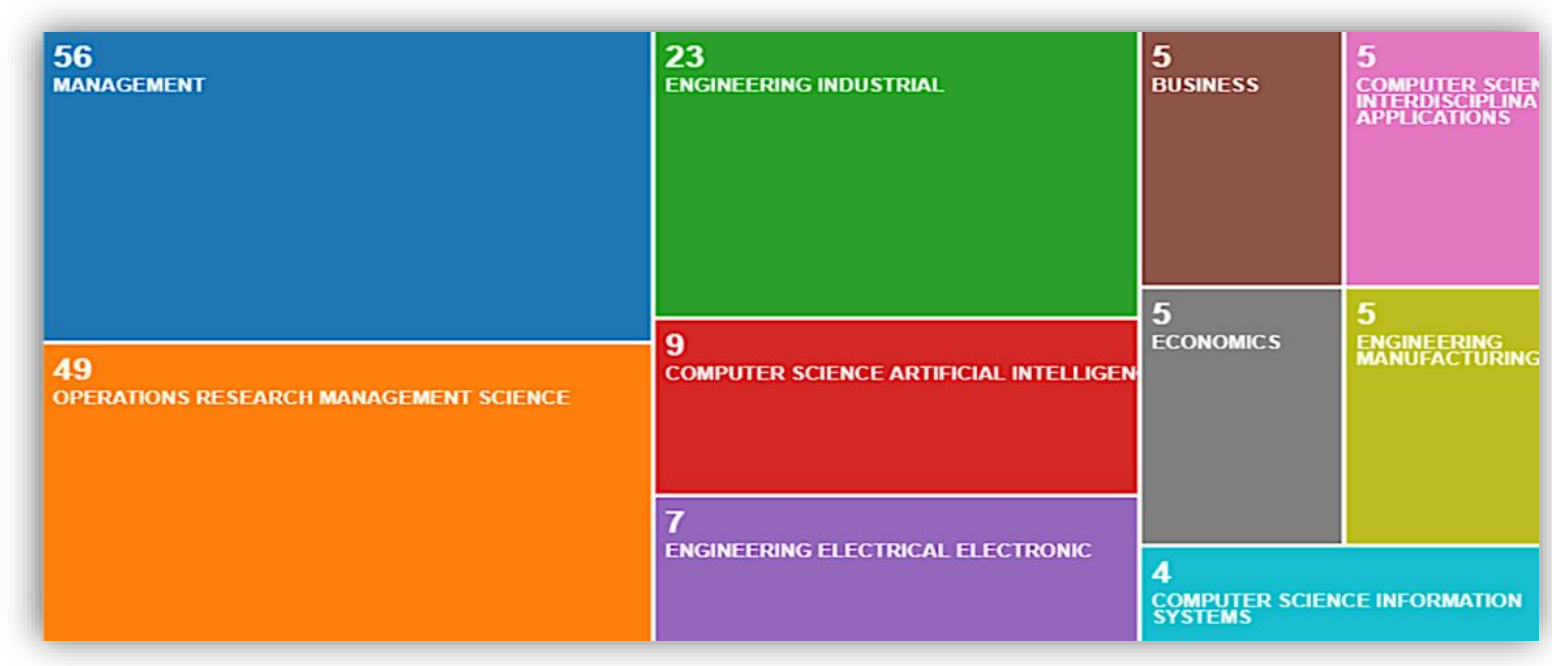

Fonte: WoS.

Ao analisar estes resultados iniciais é possível verificar a ocorrência de uma interdisciplinaridade na utilização do Analytic Hierarchy Process (AHP), fato que pode ser comprovado ao se verificar o quantitativo de 168 artigos por área, algo que ultrapassa em $54,12 \%$ o total de artigo encontrados inicialmente. Outro importante achado é a indicação do emprego em trabalhos das áreas de Ciências Sociais Aplicadas (66) e Engenharias (102), denotando o uso diversificado de critérios para apoio a tomada de decisão concentrado em três continentes (Figura 5).

Figura 5. Incidência de publicações das 10 principais instituições com registros na busca

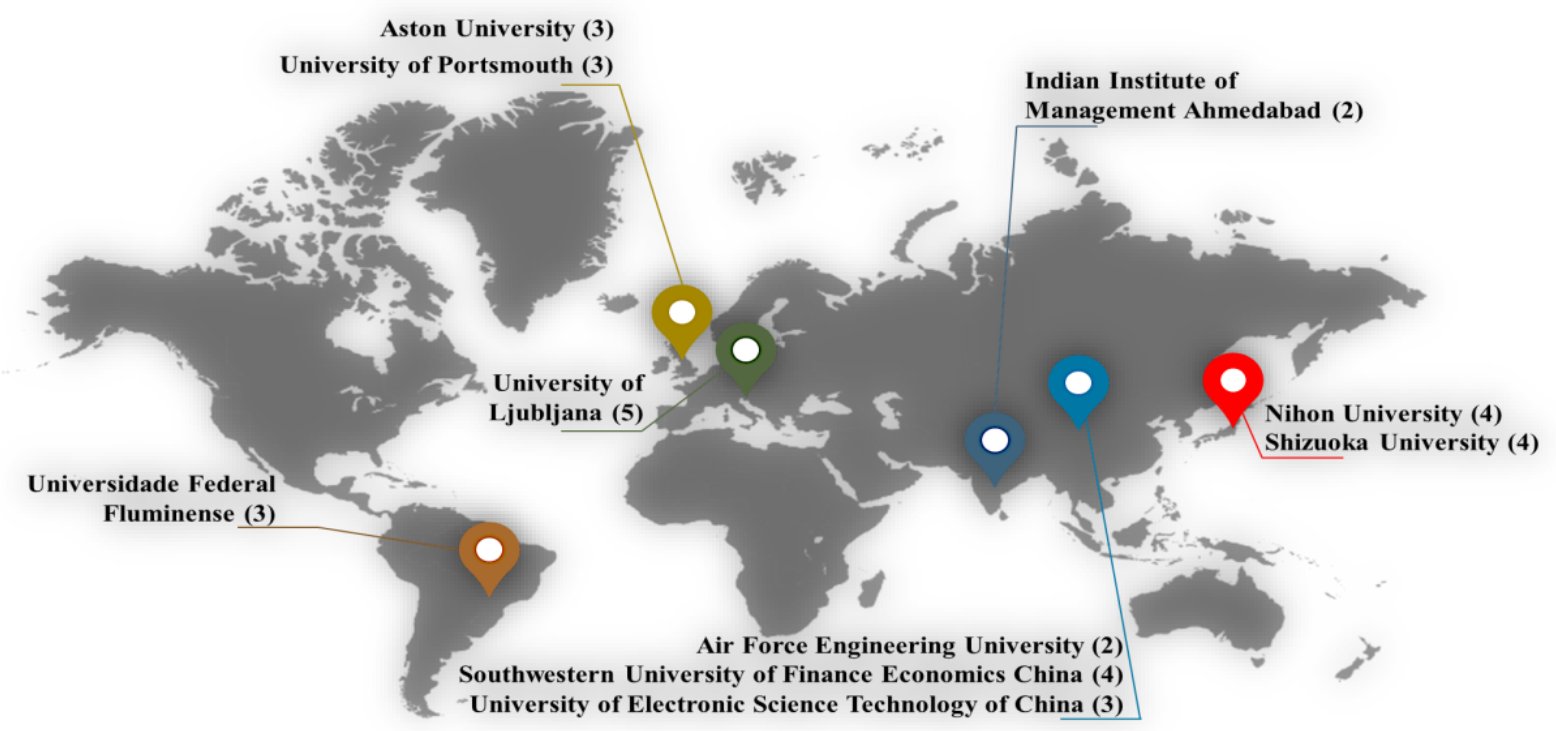

Fonte: WoS.

Assim, após realização de triagem e verificação de informações vinculadas ao arcabouço formado, houve a identificação de diversas incongruências, como dados faltantes (27 artigos), duplicados (13 artigos) e, alguns registros que apresentavam ausência dos respectivos artigos (19 registros). Seguindo as indicações de Santos et al. (2019), em que os metadados não só identificam e descrevem um objeto digital, como também documentam seu comportamento,

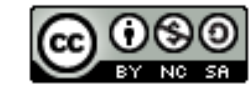

Esta obra está licenciada com uma Licença Creative Commons Atribuição-Não Comercial-Compartilha Igual 4.0 Internacional. Brazilian Journal of Production Engineering, São Mateus, Editora UFES/CEUNES/DETEC. 
Citação (APA): Gonçalves, W., Rocha, Ar. R., Zatta, F. N., \& Santos, D. V. (2021). Analytic Hierarchy Process (AHP) e a definição de atributos para apoio a tomada de decisão: uma análise bibliométrica. Brazilian Journal of Production Engineering, 7(5), 103-118.

função e uso, bem como sua relação com outros objetos digitais e como o mesmo deve ser gerenciado. Por estes motivos, as incongruências encontradas foram ajustadas, possibilitando o uso do arcabouço (109 artigos) no estabelecimento do mapeamento cientométrico (quarta etapa).

Desta forma, a primeira mineração de dados feita ao examinar o conteúdo do banco de dados utilizando o software VOSviewer considerando a coocorrência, que verificou a incidência de palavras-chave, ou seja, artigos que continham as mesmas palavras-chave utilizadas na triagem inicial na WoS (Figura 6). Essa mineração permitiu visualizar a existência da utilização híbrida do AHP com diferentes ferramentas como a fuzzy logic que auxilia a modelagem computacional do raciocínio humano e, contribui para minimização de imprecisões, diminuição de ambiguidades, dentre outras aplicabilidades e, a swot analysis que permite entender cenários e circunstâncias, possibilitando a elaboração pontual de estratégias e ações, métodos multicritério (Preference Ranking Organization Method for Enrichment Evaluation - PROMETHEE; Technique for Order Preference by Similarity to Ideal Solutions - TOPSIS), Data Envelopment Analysis (DEA), Geographic Information Systems (GIS) e Delphi method.

Figura 6. Mapa de rede mostrando relações entre várias coocorrências

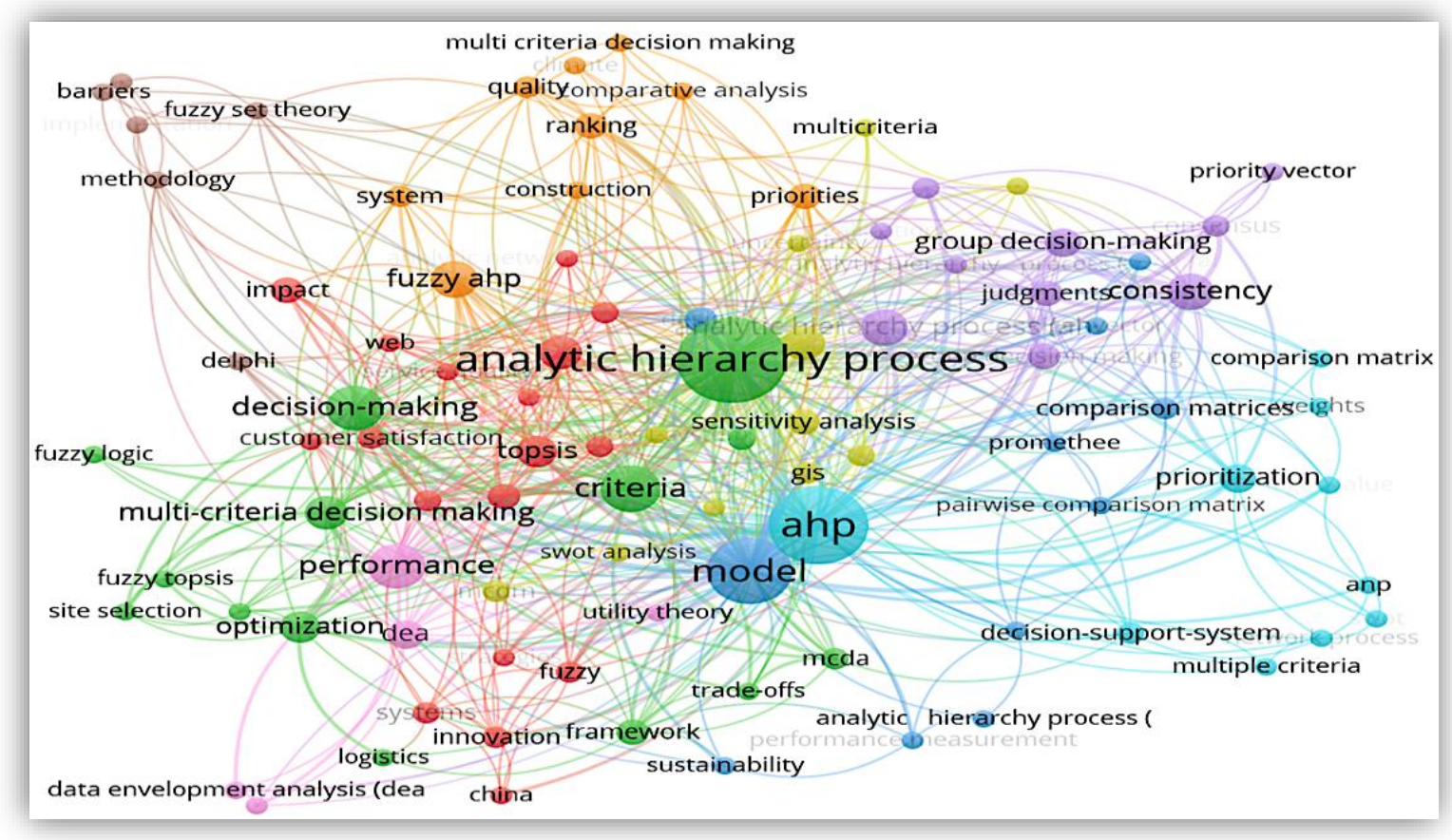

Fonte: Autores (2021), VOSviewer.

Ainda foi verificada a existência de 571 palavras-chave que apresentaram polarizações específicas no período 2014-2019, sendo que, 94 destas possuem pelo menos duas incidências, enfatizando assim a importância do AHP para apoio a tomada de decisão na gestão de atividades e operações. Dentro desse contexto, ainda foi observado que no período 2014-2018 houve uma evidência de publicações relacionadas ao uso híbrido do AHP e outras ferramentas e técnicas com foco no auxílio à resolução de problemas que envolvem o tema performance (quinta etapa). De acordo com a mineração de dados realizada, 8 dos 10 artigos mais citados foram publicados por periódicos de alta classificação (Tabela 2), sendo os 3 artigos mais citados publicados por 

atributos para apoio a tomada de decisão: uma análise bibliométrica. Brazilian Journal of Production Engineering, 7(5), $103-118$.

Kou e Lin (2014) com 219 citações; Awasthi, Govindan e Gold (2018) com 158 citações e, Kubler, Robert, Derigent, Voisin e Le Traon (2016) com 152 citações. Com relação ao impacto destes artigos mais citados é importante notar que, a maioria dos periódicos (Tabela 1) possui elevado número de citações, considerando os documentos publicados nos três anos anteriores a 2020, exceto in press os quais não agregam valor ao periódico pelo indicador CiteScore. Contudo, embora apresentem contundente relevância acadêmica estes periódicos são sediados unicamente na Europa, suscitando uma possível hegemonia relacionada ao tema da pesquisa.

Tabela 1. Principais periódicos indexados mapeados na mineração de dados

\begin{tabular}{|c|c|c|c|c|c|c|c|}
\hline Autores & Periódicos & País ${ }^{1}$ & Categoria JCR & CiteScore $^{2}$ & $\mathrm{JCR}^{2}$ & $\begin{array}{c}\text { H- } \\
\text { Index }\end{array}$ & Artigos $^{3}$ \\
\hline $\begin{array}{l}\text { Ho, Dey e } \\
\text { Lockström } \\
(2011)\end{array}$ & $\begin{array}{l}\text { Supply Chain } \\
\text { Management } \\
\text { (SCM) }\end{array}$ & UKG & $\begin{array}{c}\text { Business (17 de 153; Q1); } \\
\text { Management (17 de 226; Q1). }\end{array}$ & 9,3 & 9,012 & 115 & 16141 \\
\hline $\begin{array}{c}\text { Kubler } \text { et al. } \\
\quad(2016)\end{array}$ & $\begin{array}{l}\text { Expert Systems } \\
\text { with Applications } \\
\text { (ESA) }\end{array}$ & UKG & $\begin{array}{c}\text { Computer Science, Artificial } \\
\text { Intelligence (23 de 140; Q1); } \\
\text { Engineering, Electrical \& } \\
\text { Electronic (24 de 273; Q1); } \\
\text { Operations Research \& } \\
\text { Management Science (8 de 84; } \\
\text { Q1). }\end{array}$ & 12,7 & 6,954 & 207 & 2398 \\
\hline $\begin{array}{l}\text { Kou e Lin } \\
\text { (2014) }\end{array}$ & $\begin{array}{l}\text { European Journal } \\
\text { of Operational } \\
\text { Research (EJR) }\end{array}$ & NLD & $\begin{array}{c}\text { Operations Research \& } \\
\text { Management Science }(15 \mathrm{de} \\
\text { 84; Q1). }\end{array}$ & 9,5 & 5,334 & 260 & 2156 \\
\hline $\begin{array}{l}\text { Yahya e } \\
\text { Kingsman } \\
(1999)\end{array}$ & $\begin{array}{c}\text { Journal of the } \\
\text { Operational } \\
\text { Research Society } \\
\text { (JOS) }\end{array}$ & UKG & $\begin{array}{c}\text { Management (155 de 226; } \\
\text { Q3); Operations Research \& } \\
\text { Management Science (34 de } \\
\text { 84; Q2). }\end{array}$ & 4,1 & 2,86 & 108 & 1231 \\
\hline $\begin{array}{l}\text { Awasthi, } \\
\text { Govindan e } \\
\text { Gold (2018) }\end{array}$ & $\begin{array}{l}\text { International } \\
\text { Journal of } \\
\text { Production } \\
\text { Economics (IJE) }\end{array}$ & NLD & $\begin{array}{c}\text { Engineering, Industrial (5 de } \\
\text { 49; Q1); Engineering, } \\
\text { Manufacturing (5 de 50; Q1) } \\
\text { Operations Research \& } \\
\text { Management Science (3 de 84; } \\
\text { Q1). }\end{array}$ & 12,2 & 7,885 & 185 & 1066 \\
\hline $\begin{array}{l}\text { Dey e Cheffi } \\
\text { (2013) }\end{array}$ & $\begin{array}{c}\text { Production } \\
\text { Planning \& } \\
\text { Control (PPC) }\end{array}$ & UKG & $\begin{array}{c}\text { Engineering, Industrial (7 de } \\
\text { 49; Q1); Engineering, } \\
\text { Manufacturing (9 de 50; Q1); } \\
\text { Operations Research \& } \\
\text { Management Science (6 de 84; } \\
\text { Q1). }\end{array}$ & 8,2 & 7,044 & 76 & 884 \\
\hline $\begin{array}{l}\text { Podgórski } \\
\text { (2015) }\end{array}$ & $\begin{array}{l}\text { Safety Science } \\
\text { (SS) }\end{array}$ & NLD & $\begin{array}{c}\text { Engineering, Industrial (15 de } \\
\text { 49; Q2); Operations Research } \\
\& \text { Management Science (20 de } \\
\text { 84; Q1). }\end{array}$ & 7,8 & 4,877 & 111 & 753 \\
\hline $\begin{array}{l}\text { Mosadeghi, } \\
\text { Warnken, } \\
\text { Tomlinson e } \\
\text { Mirfenderesk } \\
\quad \text { (2015) }\end{array}$ & $\begin{array}{l}\text { Computers, } \\
\text { Environment and } \\
\text { Urban Systems } \\
\text { (CES) }\end{array}$ & UKG & $\begin{array}{l}\text { Environmental Studies (26 de } \\
\text { 125; Q1); Geography (8 de 85; } \\
\text { Q1); Regional \& Urban } \\
\text { Planning (7 de 40; Q1). }\end{array}$ & 10,1 & 5,324 & 92 & 275 \\
\hline $\begin{array}{c}\text { Ossadnik, } \\
\text { Schinke e } \\
\text { Kaspar } \\
(2016)\end{array}$ & $\begin{array}{l}\text { Group Decision } \\
\text { and Negotiation } \\
\text { (GDN) }\end{array}$ & NLD & $\begin{array}{c}\text { Management (163 de 226; } \\
\text { Q3); Social Sciences, } \\
\text { Interdisciplinary (31 de 110; } \\
\text { Q2). }\end{array}$ & 4,1 & 2,648 & 60 & 204 \\
\hline $\begin{array}{l}\text { Fouladgar, } \\
\text { Yazdani- } \\
\text { Chamzini, } \\
\text { Lashgari, } \\
\text { Zavadskas e } \\
\text { Turskis } \\
\text { (2012) } \\
\end{array}$ & $\begin{array}{l}\text { International } \\
\text { Journal of } \\
\text { Strategic } \\
\text { Property } \\
\text { Management } \\
\text { (IJM) }\end{array}$ & LTU & $\begin{array}{c}\text { Management (184 de 226; } \\
\text { Q4). }\end{array}$ & 3,6 & 2,093 & 30 & 102 \\
\hline
\end{tabular}

${ }^{1}$ Legenda: Lithuania (LTU); Netherlands (NLD) e United Kingdom (UKG). ${ }^{2}$ Ano base 2020. ${ }^{3}$ Artigos publicados até junho de 2021 que possuem em seu título ou resumo as palavras Analytic Hierarchy Process ou AHP 
Durante a mineração foi possível identificar dois artigos que realizaram uma revisão e outro análise bibliométrica da literatura, não havendo aplicações quanto ao uso do AHP para gestão de atividades e operações, Kubler et al. (2016) e Ossadnik et al. (2016), por esse motivo esses artigos foram retirados do prosseguimento das análises. Outro aspecto importante da mineração realizada foi verificar a percepção quanto a credibilidade da comunidade acadêmica sobre os periódicos das principais publicações analisadas (Tabela 2). Desta forma, foi possível mapear a amplitude e abrangência de parcerias realizadas ao longo de 21 anos (Figura 7).

Figura 7. Panorama evolutivo do arcabouço (principais periódicos indexados mapeados)

(a) Citações por documento (últimos 4 anos)

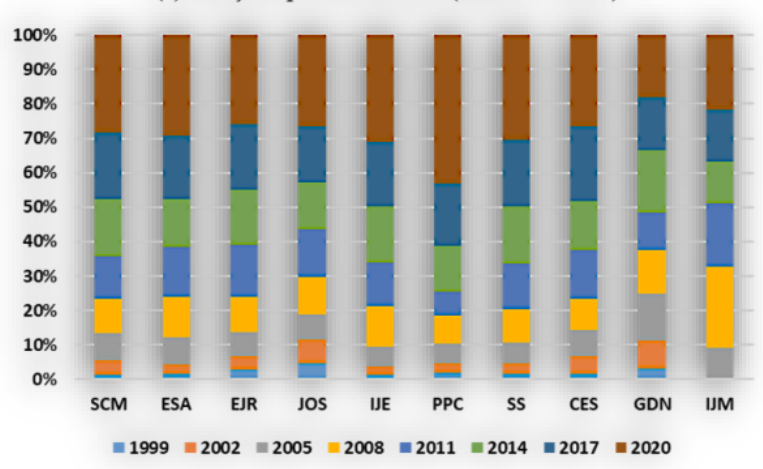

(b) Colaboração Internacional

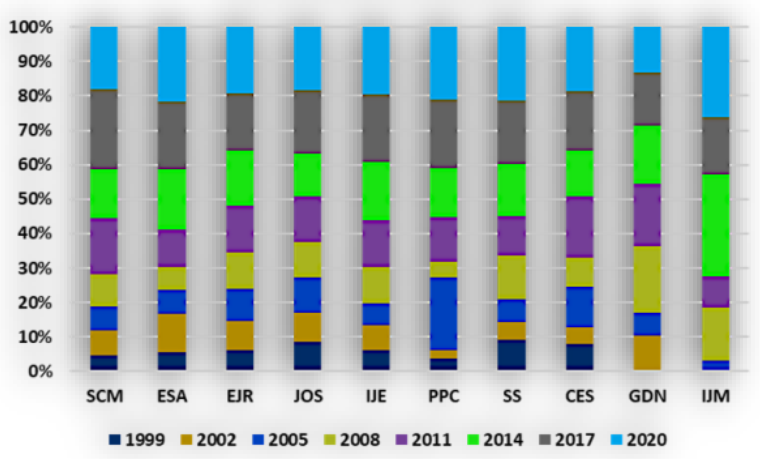

Fonte: WoS.

Seguindo a abordagem metodológica proposta, ao examinar um estrato de performance no tempo (Figura 7), é possível identificar quais destes periódicos têm promovido tendências e questões relevantes frente a academia, além de, difundirem conhecimento entre diferentes grupos e nacionalidades. Dentro desse aspecto, a Figura 8 expande o entendimento cientométrico do arcabouço analisado e, com isso, possibilita triangular informações que permitem entender o desempenho em termos destes periódicos, além de observar a ocorrência da expansão do conhecimento envolvendo colaborações internacionais, as quais podem explicar o volume de acessos e citações do arcabouço.

Figura 8. Mapa cientométrico do arcabouço analisado

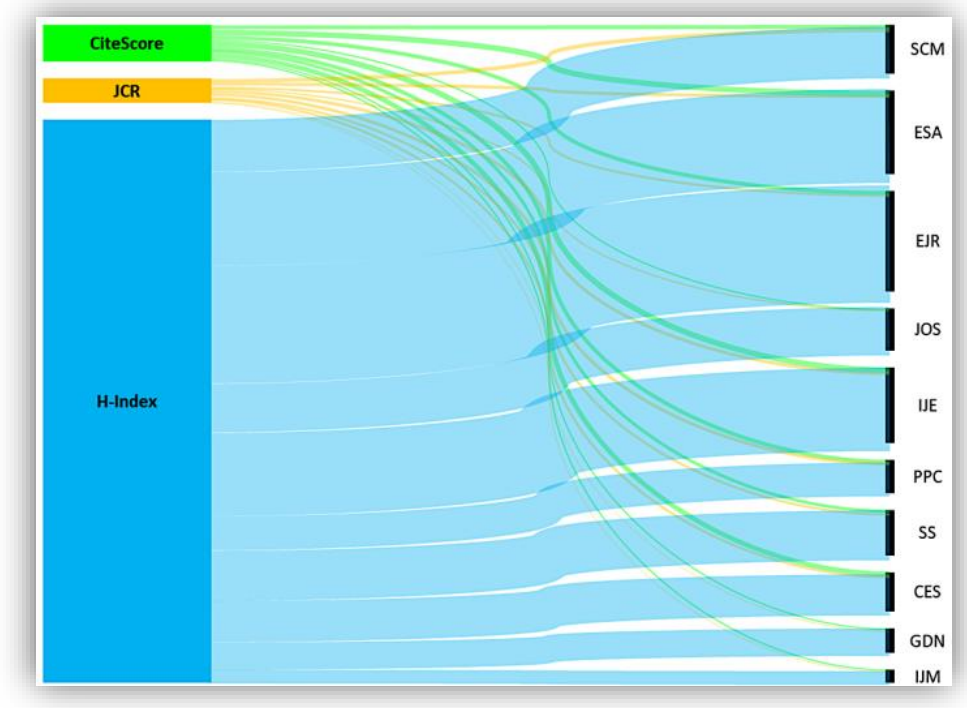

Fonte: Autores, 2021. 
Citação (APA): Gonçalves, W., Rocha, Ar. R., Zatta, F. N., \& Santos, D. V. (2021). Analytic Hierarchy Process (AHP) e a definição de atributos para apoio a tomada de decisão: uma análise bibliométrica. Brazilian Journal of Production Engineering, 7(5), $103-118$.

Complementando os achados das Figuras 7 e 8 foi elaborado compêndio das literaturas do arcabouço (Tabela 2).

Tabela 2. Estrato final de autores

\begin{tabular}{ccc}
\hline Autores & Afiliação & Objetivo(s) \\
\hline & & \\
& University of Finance & Proposição de um \\
Kou e Lin & and Economics $($ China $)$ e & método de maximização \\
(2014) & Yangtze Normal & de cosseno baseado em \\
& University (China). & medida de similaridade.
\end{tabular}

Parâmetros e contribuições para definição de atributos

Uso de um método de maximização de cosseno baseado em medida de similaridade, que maximiza a soma do cosseno do ângulo entre o vetor de prioridade e cada vetor coluna de uma matriz de comparação paritária. Com isso, os resultados mostraram além da flexibilidade e eficiência do método proposto que, combinar diferentes métodos de priorização deve ser considerado como uma nova direção.

Testagem de uma estrutura híbrida baseada na abordagem fuzzy AHP-VIKOR, integrada por meio de duas etapas, sendo os critérios definidos segundo a literatura existente e, posteriormente restringidos com a assistência de especialistas de campo e tomadores de decisão.

Seleção de fornecedores por meio de abordagem integrada fuzzy AHPVIKOR.

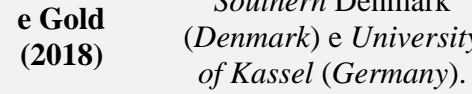

Mosadeghi

et al.

(2015)

Griffith University (Australia) e Gold Coast City Council (Australia).
Mostrar como a tomada de decisão pode fornecer uma visão sobre a extensão espacial de alternativas.

Conjectura sobre um processo para avaliar fornecedores.

Seleção de principais indicadores para medir o desempenho operacional

$\begin{array}{ll}\text { Podgórski } & \text { National Research } \\ \text { (2015) } & \text { Institute (Poland) }\end{array}$

Dey e

Cheffi

(2013)

Aston University (United
Kingdom) e Rouen
Business School
(France).

Desenvolver um framework estruturado analítico para medir o desempenho.

Propor uma abordagem QFD-AHP para elevar a eficácia das decisões de abastecimento.

Aplicação Fuzzy-AHP nos conceitos de COPRAS para avaliar classificacões e pesos.

Tehran (Iran) e Vilnius

University (Lithuania)
Emprego de categorização para definição de critérios e classificações, as quais subsidiaram a

elaboração dos subcritérios. Além disso, é apresentado como a tomada de decisão pode ser usada não apenas para classificar a prioridade de opções e realizar a análise de cenário, mas também para fornecer uma visão sobre ampliada das alternativas

Revisão sistemática da literatura e de documentos técnicos para posterior mineração de dados visando a seleção dos atributos mais significantes.

Serventia de revisão da literatura sobre principais atributos sobre indicadores chave de performance, os quais forneceram uma base lógica.

As bases teóricas do trabalho combinam e reconciliam três áreas principais: gestão da cadeia de suprimentos, gestão ambiental e medição de desempenho, tendo por foco o levantamento de atributos para auxílio à monitoração de desempenho da cadeia de abastecimento verde. $\mathrm{Na}$ abordagem, o QFD é usado para traduzir os requisitos das partes interessadas da empresa em

vários fatores de avaliação para seleção de

fornecedores, que são usados para avaliar os fornecedores. O AHP é utilizado para determinar a importância dos fatores de avaliação e preferência de cada fornecedor com relação a cada critério de seleção.

Revisão sistemática da literatura e colaboração de experts visando a seleção dos atributos mais significantes.

Fonte: Produção do próprio autor. Legenda: VIKOR - VlseKriterijumska Optimizacija I Kompromisno Resenje. QFD - Quality Function Deployment. COPRAS - COmplex PRoportional Assessment.

Trabalhos que envolvem consulta a literatura - quais seja acadêmica ou técnica (Tabela 2), ao longo do tempo, tem mantido a essência da proposta inicial do AHP (Saaty, 1991), em que, atributos apontados pela academia devam ser associados às diferentes opiniões de stakeholders e especialistas. Outro aspecto importante é o uso híbrido de técnicas, métodos, ferramentas, e outros dispositivos que auxiliem na elucidação inicial de operacionalização de dados, assim 
como, na complementação da compreensão dos resultados apresentados. Como por exemplo, o uso da abordagem integrada fuzzy AHP-VIKOR, a qual permite uma depuração de opiniões e um esclarecimento ampliado de respostas (sexta etapa).

\section{CONSIDERAÇÕES FINAIS}

A análise bibliométrica é uma ferramenta efetiva para obter uma visão holística de uma área específica da pesquisa ou de um periódico. Por esse motivo, essas análises podem ser de elevado interesse para diferentes interessados em uma determinada área de pesquisa e, com isso, além de elucidar importantes achados, é possível identificar tendências e questões relevantes. Neste trabalho, estas análises fornecem a possibilidade da formação de uma agenda de pesquisa e, também, diretrizes de investigação a serem desenvolvidas no futuro.

Durante a operacionalização da abordagem metodológica foi constatado que diversos trabalhos apresentaram o Analytic Hierarchy Process (AHP) como uma teoria. No entanto, essa designação tem suas origens ligadas ao Prof. Thomas Lorie Saaty, que em 1971 - enquanto trabalhava no Departamento de Defesa dos Estados Unidos da América, elaborou um planejamento eventual (Saaty, 1991). E, nos anos posteriores, após o entendimento das potencialidades dessa teoria, vislumbrando sua replicabilidade, o método AHP foi elaborado a fim de contribuir com a resolução de problemas e, sua conceitualização e estruturação a partir do entendimento da mente humana.

Além disso, a visualização da cocitação, países e universidades, e a coocorrência das palavraschave dos autores nos ajudaram a descobrir padrões de difusão do conhecimento entre as comunidades interconectadas. Outrossim, a análise da coocorrência de palavras-chave mostrou uma tendência de conceitos-chave durante 2014-2019. As palavras-chave mais utilizadas estão relacionadas ao esclarecimento e refinação de dados e, também, voltadas a consolidação da compreensão de resultados.

Uma limitação deste trabalho diz respeito à duração de nossa análise, devido ela variar entre 1970 e 2020; os resultados podem mudar no futuro, pois acreditamos que a natureza mutante do método AHP pode trazer novos assuntos, conceitos e metodologias que podem originar mudanças significativas aos resultados apresentados acima.

Por fim, como as análises se basearam em dados coletados na base WoS, as limitações associadas à esta base também podem ser transferidas para este trabalho. De tal modo, os resultados deste trabalho evidenciam que há um amplo campo de estudo a ser explorado sobre o tema pesquisado. Por essa razão, sugere-se que análises futuras devem ser conduzidas com dados obtidos de fontes múltiplas, ampliando as buscas adicionando outros rótulos de campo.

\section{AgradeCimentos}

Agradecemos ao apoio financeiro da Coordenação de Aperfeiçoamento de Pessoal de Nível Superior - Brasil (CAPES) e ao Laboratório de Pesquisa Operacional, Logística e Transporte (POLT) da Universidade Federal do Espírito Santo (UFES) / Centro Universitário Norte do Espírito Santo (CEUNES) pelo apoio acadêmico e técnico na concepção e desenvolvimento deste trabalho.

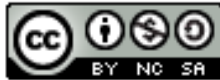


Citação (APA): Gonçalves, W., Rocha, Ar. R., Zatta, F. N., \& Santos, D. V. (2021). Analytic Hierarchy Process (AHP) e a definição de atributos para apoio a tomada de decisão: uma análise bibliométrica. Brazilian Journal of Production Engineering, 7(5), 103-118.

\section{REFERÊNCIAS}

Awasthi, A., Govindan, K., \& Gold, S. (2018). Multi-tier sustainable global supplier selection using a fuzzy AHP-VIKOR based approach. International Journal of Production Economics, 195, 106-117. https://doi.org/10.1016/j.ijpe.2017.10.013.

Bruno, G., Esposito, E., Genovese, A., \& Passaro, R. (2012). AHP-based approaches for supplier evaluation: problems and perspectives. Journal of Purchasing \& Supply Management, 18(3), 159-172. https://doi.org/10.1016/j.pursup.2012.05.001.

Cebrino, J., \& Portero De La Cruz, S. (2020). A worldwide bibliometric analysis of published literature on workplace violence in healthcare personnel. PLoS one, 15(11), e0242781. https://doi.org/10.1371/journal.pone.0242781.

Chan, F. T. S., \& Chan, H. K. (2004). Development of the supplier selection model - a case study in the advanced technology industry. Proceedings of the Institution of Mechanical Engineers Part B - Journal of Engineering Manufacture, 4(3), 1807-1824. https://doi.org/10.1177/095440540421801213.

Demuner, M. T., Zatta, F. N., Freitas, R. R., \& Goncalves, W. (2020). Multidimensional approach to elaborate strategic map in small businesses through the integration BSC-AHP. Brazilian Journal of Production Engineering, 6(3), 57-74. Recuperado de https://www.periodicos.ufes.br/bjpe/article/view/29290.

Dey, P. K., \& Cheffi, W. (2013). Green supply chain performance measurement using the analytic hierarchy process: a comparative analysis of manufacturing organisations. Production Planning \& Control, 24(8-9), 702-720. https://doi.org/10.1080/09537287.2012.666859.

Fouladgar, M. M., Yazdani-Chamzini, A., Lashgari, A., Zavadskas, E. K., \& Turskis, Z. (2012). Maintenance strategy selection using AHP and COPRAS under fuzzy environment. International journal of strategic property management, 16(1), 85-104. https://doi.org/10.3846/1648715X.2012.666657.

Friendly, A. (2019). The contradictions of participatory planning: Reflections on the role of politics in urban development in Niterói, Brazil. Journal of Urban Affairs, 41(7), 910-929. https://doi.org/10.1080/07352166.2019.1569468.

Gonçalves, W. (2016). Integração de Técnicas de Análise Multivariada e Método Multicritério para Localização de Centros de Distribuição (Tese de doutorado). Universidade Metodista de Piracicaba, Santa Bárbara D'Oeste, SP, Brasil. Recuperado de https://iepapp.unimep.br/biblioteca_digital/pdfs/docs/18112016_160918_wellingtongoncalves _ok.pdf.

Gora, A. A. (2019). The Link Between Decision Making Process and Performance: A Bibliometric Analysis. Management and Economics Review, 4(2), 177-191. Recuperado de http://mer.ase.ro/files/2019_2/4-8.pdf.

Ho, W., Dey, P. K., \& Lockström, M. (2011). Strategic sourcing: a combined QFD and AHP approach in manufacturing. Supply Chain Management: An International Journal, 16(6), 446461. https://doi.org/10.1108/13598541111171093.

Ho, W., \& Ma, X. (2018). The state-of-the-art integrations and applications of the analytic hierarchy process. European Journal of Operational Research, 267(2), 399-414, https://doi.org/10.1016/j.ejor.2017.09.007. 

atributos para apoio a tomada de decisão: uma análise bibliométrica. Brazilian Journal of Production Engineering, 7(5), $103-118$.

Kou, G., \& Lin, C. (2014). A cosine maximization method for the priority vector derivation in AHP. European Journal of Operational Research, 235(1), 225-232, https://doi.org/10.1016/j.ejor.2013.10.019.

Kubler, S., Robert, J., Derigent, W., Voisin, A., \& Le Traon, Y. (2016). A state-of the-art survey $\&$ testbed of fuzzy AHP (FAHP) applications. Expert Systems with Applications, 65, 398-422. https://doi.org/10.1016/j.eswa.2016.08.064.

Martins, C. L., López, H. M. L., Almeida, A. T., Almeida, J. A., \& Oliveira Bortoluzzi, M. B. (2017). An MCDM project portfolio web-based DSS for sustainable strategic decision making in an electricity company. Industrial Management \& Data Systems, 117(7), 1362-1375. https://doi.org/10.1108/IMDS-09-2016-0412.

Merigó, J. M., \& Yang, J. B. (2017). A bibliometric analysis of operations research and management science. Omega, 73, 37-48. https://doi.org/10.1016/j.omega.2016.12.004.

Mosadeghi, R., Warnken, J., Tomlinson, R., \& Mirfenderesk, H. (2015). Comparison of FuzzyAHP and AHP in a spatial multi-criteria decision making model for urban land-use planning. Computers, Environment and Urban Systems, 49, 54-65. https://doi.org/10.1016/j.compenvurbsys.2014.10.001.

Muralidharan, C., Anantharaman, N., \& Deshmukh, S. G. (2002). A multi-criteria group decisionmaking model for supplier rating. Journal of supply chain management, 38(3), 22-33. https://doi.org/10.1111/j.1745-493X.2002.tb00140.x.

Ossadnik, W., Schinke, S., \& Kaspar, R. H. (2016). Group aggregation techniques for analytic hierarchy process and analytic network process: a comparative analysis. Group Decision and Negotiation, 25(2), 421-457. https://doi.org/10.1007/s10726-015-9448-4.

Petruni, A., Giagloglou, E., Douglas, E., Geng, J., Leva, M. C., \& Demichela, M. (2019). Applying Analytic Hierarchy Process (AHP) to choose a human factors technique: Choosing the suitable Human Reliability Analysis technique for the automotive industry. Safety Science, 119, 229-239. https://doi.org/10.1016/j.ssci.2017.05.007.

Podgórski, D. (2015). Measuring operational performance of OSH management system-A demonstration of AHP-based selection of leading key performance indicators. Safety science, 73, 146-166. https://doi.org/10.1016/j.ssci.2014.11.018.

Qaiser, F. H., Ahmed, K., Sykora, M., Choudhary, A., \& Simpson, M. (2017). Decision support systems for sustainable logistics: a review and bibliometric analysis. Industrial Management \& Data Systems, 117(7), 1376-1388. https://doi.org/10.1108/IMDS-09-2016-0410.

Saaty, T. L. (1977). A Scaling Method for Priorities in Hierarchical Structures. Journal of Mathematical Psychology, 15, 234-281. https://doi.org/10.1016/0022-2496(77)90033-5. (1991). Método de análise hierárquica. São Paulo: McGraw-Hill, Makron Books. (2008). Decision making with the analytic hierarchy process. International journal of services sciences, 1(1), 83-98. https://doi.org/10.1504/IJSSci.2008.017590.

Sánchez, A. D., Del Río, M. D. L. C., \& García, J. Á. (2017). Bibliometric analysis of publications on wine tourism in the databases Scopus and WoS. European Research on $\begin{array}{llll}\text { Management and Business } & \text { 8conomics, }\end{array}$ https://doi.org/10.1016/j.iedeen.2016.02.001.

Santos, P. H., Neves, S. M., Sant'Anna, D. O., Oliveira, C. H., \& Carvalho, H. D. (2019). The analytic hierarchy process supporting decision making for sustainable development: An 

atributos para apoio a tomada de decisão: uma análise bibliométrica. Brazilian Journal of Production Engineering, 7(5), $103-118$.

overview of applications. Journal of cleaner production, 212, 119-138. https://doi.org/10.1016/j.jclepro.2018.11.270.

Webb, M. J. (2018). Estimating Uncertainty Attributable to Inconsistent Pairwise Comparisons in the Analytic Hierarchy Process (AHP) - (Tese de doutorado). George Washington University, Washington, 2018.

Yadav, V., \& Sharma, M. K. (2016). Multi-criteria supplier selection model using the analytic hierarchy process approach. Journal of Modelling in Management, 11(1), 326-354. https://doi.org/10.1108/JM2-06-2014-0052.

Yahya, S., \& Kingsman, B. (1999). Vendor rating for an entrepreneur development programme: a case study using the analytic hierarchy process method. Journal of the Operational Research Society, 50(9), 916-930, https://doi.org/10.1057/palgrave.jors.2600797.

Zatta, F. N., Mattos, A. L., Oliveira, R. R., Freitas, R. R., \& Gonçalves, W. (2019). Aplicação do Analytic Hierarchy Process na escolha de planos de saúde. Research, Society and Development, 8(1), p. e1881532, https://doi.org/10.33448/rsd-v8i1.532.

Zhou, S., \& Yang, P. (2020). Risk management in distributed wind energy implementing Analytic Hierarchy Process. Renewable Energy, 150, 616-623. https://doi.org/10.1016/j.renene.2019.12.125. 